\title{
Dynamic Resonance in the High-Q and Near-Monochromatic Regime
}

\author{
Gayan S. Abeynanda \\ Department of Mathematics \\ Louisiana State University \\ Baton Rouge, LA 70803, USA \\ Email: gabeyn1@1su.edu
}

\author{
Stephen P. Shipman \\ Department of Mathematics \\ Louisiana State University \\ Baton Rouge, LA 70803, USA \\ Email: shipman@math.lsu.edu
}

\begin{abstract}
This communication investigates the sensitive behavior of dynamic resonant scattering when an extended system is weakly coupled to a resonator (high-Q) and the source field is nearly monochromatic but of finite energy. We analyze a specific model that incorporates certain universal features of resonance. It is a resonant modification of the Lamb model for the interaction between an oscillator and a continuum. This resonant Lamb model incorporates a two-part scatterer attached to an infinite string with continuous spectrum. The non-resonant part of the scatterer is associated with direct scattering; and the resonant part-an oscillator coupled weakly to the stringis associated with field amplification and delayed scattering. A sharp anomaly in the transmission coefficient occurs when the source frequency is within $\gamma^{2}$ of the resonant frequency (where $\gamma$ is the coupling constant and the Q-factor is of order $\gamma^{-2}$ ); this anomaly is mollified as the source spreads spectrally. Resonant amplification occurs when the source frequency is within $\gamma$ of the resonant frequency. The amount of amplification depends on the relationships between the spectral width $\gamma^{2}$ of the transmission resonance, the spectral width $\sigma$ of the source field, and the difference between the source frequency and the resonant frequency.
\end{abstract}

\section{DYNAMiC RESONANCE}

Photonic devices often require the precise tuning of certain features of electromagnetic resonances, such as the central frequency of the resonance, its spectral width or Q-factor (quality factor), and its asymmetry. Mathematical analysis of these features often assumes that a device is operating in the ideal monochromatic regime. But finite-time operation causes frequency broadening and can significantly alter the crisp frequency-domain picture. It also raises additional questions about the dynamical nature of resonance, such as the partition of energy into direct and resonant scattering channels and the build-up and decay time of resonant fields. Often a coupledmode analysis is invoked. Such models isolate phenomenological component "modes" of a resonant scattering process and can yield good predictive models; see [1] for a general theory and [2], [3] for a coupled-mode theory for photonic resonators.

The present study analyzes resonant scattering of a system, wherein the modes of the system are not postulated but arise from the model itself. The goals include evaluating the accuracy of a coupled-mode theory for a system by comparing it to the true dynamics of the system; and quantifying the delicate behavior of resonance features in the simultaneous high-Q and near-monochromatic regimes. This paper confines itself to the latter objective. High Q-factor means high resonant amplification and slow energy decay from the resonator within a narrow frequency band; and the near-monochromatic regime refers to operation under a well-defined central, or carrier, frequency that is tapered in time and space.

The scattering characteristics of a photonic device operating at resonance are very sensitive to parameters of the structure and the source field. Specific devices are analyzed with a combination of mathematical and numerical methods, and the analysis often treats the resonant scattering of Gaussian beams and pulses and how it depends on the angle of incidence; see [4], [5], for example. The model we present in this short communication is not complicated enough to address aspects of angle of incidence. Our aim is to rigorously address universal aspects of resonant amplification with regard to the delicate balance between the spectral widths of the resonance and the source field.

We analyze what we feel is the simplest model system that exhibits certain essential features of photonic resonance in the arbitrarily high- $\mathrm{Q}$ regime. The model is inspired by the famous "Lamb model" proposed by H. Lamb to elucidate the interaction between an extended medium and a nucleus [6]. Lamb's model consists of an infinite string coupled to a spring-mass system, in which radiation losses are felt by the mass as equivalent to instantaneous linear damping. In our modification, illustrated in Fig. 1, a point mass is attached to the string, and that point mass is in turn weakly coupled to a separate spring-mass resonator. The point mass on the string serves as the non-resonant part of the scatterer, and the springmass serves as the resonant part. Lamb-type models can serve as elucidating prototypes for diverse scattering phenomena, such as irreversibility [7], state transitions [8], gyroscopic instability [9], and nonlinear bistability [10]. A very general time-dynamic resonance theory that analyzes the intermediate and long time behavior of quantum resonant states is developed in [11].

The high-Q near-monochromatic regime involves three simultaneously small physical parameters. The constant of coupling, $\gamma$, between the point-mass and the resonator controls the spectral width of the transmission resonance (Fig. 2, top), which is of order $\gamma^{2}$, and the Q-factor, which is of order $\gamma^{-2}$. The spectrum of the resonator is centered about a frequency $\omega_{c}$. The source field will be a wave packet centered about a frequency $\bar{\omega}$ with spectral width $\sigma$. The difference between the central frequencies of the resonance and the source field 
is denoted by $\eta=\bar{\omega}-\omega_{c}$. The three parameters

$\gamma^{2}:$ spectral width of transmission resonance

$\sigma:$ spectral width of source field

$\eta$ : difference between resonant and source frequencies

are considered to be small, and we will analyze the effects of differing their relative sizes.

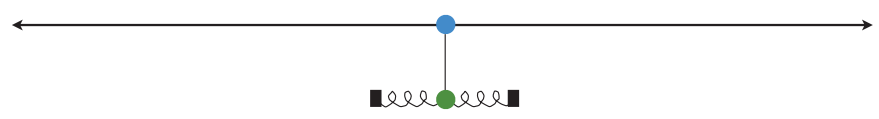

Fig. 1. A schematic depiction of a modified Lamb model that incorporates a non-resonant part, which is the point-mass on the string; and a resonant part, which is the spring-mass oscillator with arbitrarily high quality factor. The line connecting the point-mass on the string with the spring-mass oscillator (resonator) indicates an instantaneous coupling.

\section{A RESONANT LAMB MODEL}

The model of resonant scattering that we analyze is depicted schematically in Fig. 1. The ambient space is modeled by a transmission line, or string, in which disturbances $u(x, t)$ travel according to the linear wave equation. The two-part scatterer consists of a non-resonant part and a resonant part, whose displacements are denoted by $y(t)$ and $z(t)$. The equations of motion are

$$
\begin{aligned}
& u_{t t}(x, t)=c^{2} u_{x x}(x, t) \text { for } x \neq 0 \\
& \ddot{y}(t)= \beta\left(u_{x}\left(0^{+}, t\right)-u_{x}\left(0^{-}, t\right)\right)+\gamma(z(t)-y(t)) \\
& \text { with } y(t)=u(0, t) \\
& \ddot{z}(t)=-\omega_{0}^{2} z(t)+\gamma(y(t)-z(t)) .
\end{aligned}
$$

The constant $c$ is the celerity of waves in the string, $\beta$ controls the force exerted by the string on the point-mass, $\gamma$ is the coupling constant between the point-mass and the resonator, and $\omega_{0}$ is the free oscillatory frequency of the resonator.

We have devised this resonant Lamb model so that, when $\gamma=0$, the string with the point-mass is completely decoupled from the spring-mass. The free oscillation of the spring-mass is then trivially an infinite-lifetime, finite-energy state, and its frequencies $\pm \omega_{0}$ are eigenvalues for the full system that are embedded in the continuous spectrum of extended states of the string. When the coupling $\gamma$ is turned on, these eigenvalues become resonances in the lower-half complex plane with imaginary part on the order of $\gamma^{2}$. Scattering of waves experience Fano resonance near $\omega_{0}$. This kind of resonance has been analyzed in detail [2], [3], [12], [13].

\section{A. Scattering solutions for the resonant Lamb model}

We will consider source fields in the string that travel from left to right and are partly reflected by the scatterer and partly transmitted across it. Because the wave equation governs the motion of disturbances in the string, the displacement of the string during this scattering process has the form

$$
u(x, t)= \begin{cases}J(x-c t)+R(x+c t), & x \leq 0 \\ T(x-c t), & x \geq 0\end{cases}
$$

The function $J(\xi)$ is the source field, and $R(\xi)$ and $T(\xi)$ are the reflected and transmitted fields. If $J(\xi)=0$ for $\xi>\xi_{0}$, then before time $t=-\xi_{0} / c$, the excitation $J(x-c t)$ is supported completely to the left of the scatterer and is approaching it. In this case, we assume that the scatterer and the right side of the string are at rest before time $t=-\xi_{0} / c$ so that $R(\xi)=0$ for $\xi<-\xi_{0}$ and $T(\xi)=0$ for $\xi>\xi_{0}$. By moving $\xi_{0}$ to $\infty$, one can allow $J(\xi)$ to be a Gaussian, for example.

By putting $\xi=x-c t$ and setting $x=0$, one observes that the point-mass on the string experiences the source field $J(\xi)$ as a time-dependent input

$$
j(t):=J(-c t) \text {. }
$$

The Fourier-Laplace transform $\hat{\jmath}(\omega)$ of $j(t)$ is

$$
\hat{\jmath}(\omega)=\int j(t) e^{i \omega t} d t
$$

\section{B. Reduction to the scatterer}

The dynamics of the string and scatterer can be reduced to a system of ordinary differential equations for $y$ and $z$,

$$
\begin{aligned}
& \ddot{y}(t)=-\alpha \dot{y}(t)+\gamma(z(t)-y(t))+\alpha j^{\prime}(t) \\
& \ddot{z}(t)=-\omega_{0}^{2} z(t)+\gamma(y(t)-z(t)) .
\end{aligned}
$$

in which $\alpha=2 \beta / c$. With a solution to this system in hand, the transmitted and reflected fields, and therefore the full field $u(x, t)$, is obtained through

$$
\begin{aligned}
& T(\xi)=y(-\xi / c) \\
& R(\xi)=y(\xi / c)-J(-\xi) .
\end{aligned}
$$

Under the Fourier-Laplace transform, this system becomes

$$
\left[\begin{array}{cc}
\omega^{2}+i \alpha \omega-\gamma & \gamma \\
\gamma & \omega^{2}-\omega_{0}^{2}-\gamma
\end{array}\right]\left[\begin{array}{l}
\hat{y}(\omega) \\
\hat{z}(\omega)
\end{array}\right]=\left[\begin{array}{c}
i \alpha \omega \hat{\jmath}(\omega) \\
0
\end{array}\right] .
$$

The solution is

$$
\begin{aligned}
& \hat{y}(\omega)=i \alpha \frac{\omega\left(\omega^{2}-\omega_{0}^{2}-\gamma\right)}{D(\omega)} \hat{\jmath}(\omega) \\
& \hat{z}(\omega)=-i \alpha \gamma \frac{\omega}{D(\omega)} \hat{\jmath}(\omega),
\end{aligned}
$$

in which the determinant of the system is

$$
D(\omega)=\left(\omega^{2}+i \alpha \omega-\gamma\right)\left(\omega^{2}-\omega_{0}^{2}-\gamma\right)-\gamma^{2} .
$$

\section{Monochromatic scattering}

In the ideal monochromatic, or harmonic, setting, one takes the source field in the string to be a pure traveling oscillation,

$$
J(x-c t)=\exp i(\bar{\omega} x / c-\bar{\omega} t) .
$$

(by moving $\xi_{0}$ to infinity, the experiment is considered to have started an infinite amount of time ago). With $\bar{k}=\bar{\omega} / c$, one has $J(\xi)=\exp (i \bar{k} \xi)$, and the transmitted and reflected fields therefore have the oscillatory form

$$
T(\xi)=\mathfrak{t}(\bar{\omega}) e^{i \bar{k} \xi}, \quad R(\xi)=\mathfrak{r}(\bar{\omega}) e^{-i \bar{k} \xi} .
$$

The point-mass on the string experiences the input

$$
j(t)=e^{-i \bar{\omega} t}
$$


and the response in the two parts of the scatterer are the harmonic fields

$$
\begin{aligned}
y(t) & =i \alpha \frac{\bar{\omega}\left(\bar{\omega}^{2}-\omega_{0}^{2}-\gamma\right)}{D(\bar{\omega})} e^{-i \bar{\omega} t} \\
z(t) & =-i \alpha \gamma \frac{\bar{\omega}}{D(\bar{\omega})} e^{-i \bar{\omega} t}
\end{aligned}
$$

In light of the relation $T(\xi)=y(-\xi / c)$, one obtains
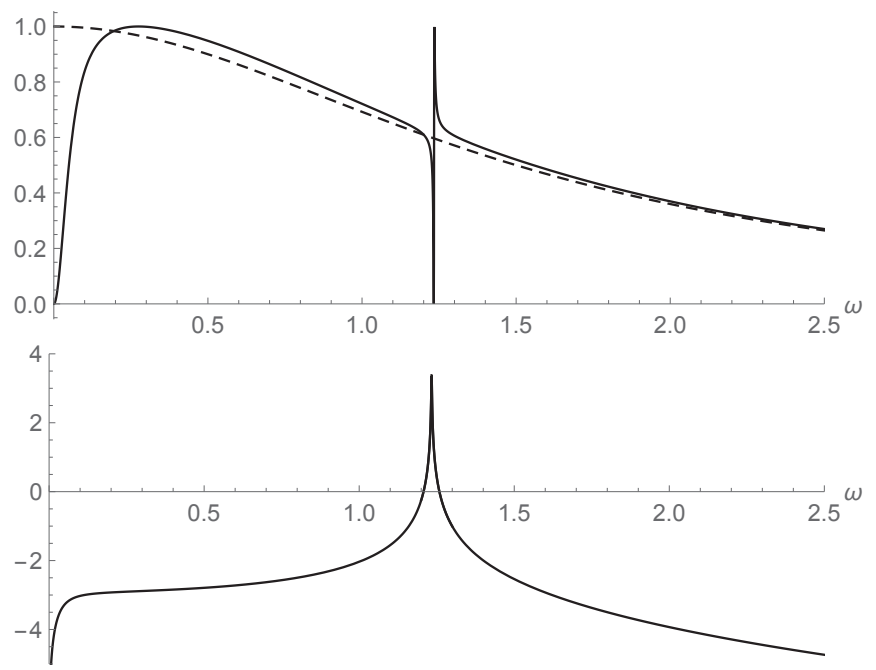

Fig. 2. The simple resonant Lamb model exhibits Fano resonance, manifest in the harmonic transmission coefficient $|\mathfrak{t}(\omega)|^{2}$ for $\gamma=0$ (dashed) and for $\gamma=0.08$ (solid); and in the resonant amplification of the resonator $\log |z|$ (below) for $\gamma=0.08$. Here, $\omega_{0}=1.2$ and $\alpha=1.5$.

$$
\mathfrak{t}(\omega)=i \alpha \frac{\omega\left(\omega^{2}-\omega_{0}^{2}-\gamma\right)}{D(\omega)},
$$

and the conservation of energy law $|\mathfrak{t}(\omega)|^{2}+|\mathfrak{r}(\omega)|^{2}=1$ implies the standard result

$$
|\mathfrak{t}(\omega)|^{2} \leq 1
$$

The quantity $|\mathfrak{t}(\omega)|^{2}$ is called the monochromatic (or harmonic) transmission coefficient. Plots of $|\mathfrak{t}(\omega)|^{2}$ for $\gamma=0$ and small nonzero $\gamma$, as well as the amplitude of the resonator $z(t)$, are shown in Fig. 2.

The sharp Fano "lineshape" and resonant amplification are well understood through analyses based on a coupled-mode theory [2], [3] and analyses based on the underlying equations of electromagnetics [12], [10]. These theories give an account of the high-Q monochromatic regime, in which the total energy of the source field is infinite.

We now would like to quantify how the spectral broadening of the source field due to having finite total energy affects these resonant features. Dealing with finite-energy sources impels one to investigate the temporal aspects of scattering.

\section{DIRECT AND RESONANT SCATTERING}

Direct and resonant scattering paths are described in [2] for resonant scattering by photonic crystal slabs. The idea is universal: Part of the energy of the source field is transmitted directly across the scatterer or reflected directly back, and part of it is coupled into the resonator, where it is resonantly amplified if the source frequency is close to that of the free resonator. The energy in the resonator then decays out, back into the string, and contributes to delayed transmission and reflection. A coupled-mode theory based on this mechanism leads to good approximations of resonant behavior of fields [3].

A clean definition of the direct and resonant parts of the scattering field is provided by the pole structure of the Fourier transform of the solution. For the resonant Lamb model, the four poles, which are the roots of the determinant $D(\omega)$, are depicted in Fig. 3. A partial-fraction decomposition of the functions multiplying $\hat{\jmath}(\omega)$ in $(1,2)$ splits the solutions $y(t)$ and $z(t)$ into four parts. Two of the poles, $\omega_{*}$ and $-\bar{\omega}_{*}$,

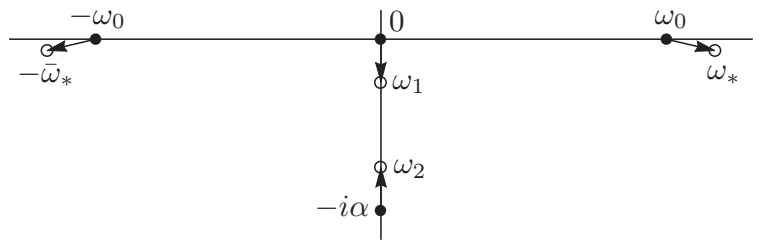

Fig. 3. The poles of $\hat{y}(\omega)$ and $\hat{z}(\omega)$ are the roots of the determinant $D(\omega)$. For zero coupling $\gamma=0$, the poles and are indicated by solid dots at $\pm \omega_{0}, 0$, and $-i \alpha$. The pole at 0 is cancelled by a root in the expressions for $\hat{y}(\omega)$ and $\hat{z}(\omega)$. For $\gamma>0$, the poles are indicated by open circles. The eigenfrequencies $\pm \omega_{0}$ move into the complex plane where they become resonances $\omega_{*}$ and $-\bar{\omega}_{*}$ with imaginary part on the order of $\gamma^{2}$.

originate from the spring-mass system and are perturbations of its characteristic frequencies $\pm \omega_{0}$; these resonances have negative imaginary part on the order of $\gamma^{2}$. The resonance $\omega_{*}$ is an analytic function of $\gamma$,

$$
\omega_{*}(\gamma)=\omega_{c}(\gamma)+i \gamma^{2} \kappa(\gamma)
$$

in which $\omega_{c}$ is the central (real) frequency of the resonance, which is detuned from $\omega_{0}$ by order $\gamma$,

$$
\omega_{c}(\gamma)=\omega_{0}+\frac{1}{2 \omega_{0}} \gamma+O\left(\gamma^{2}\right),
$$

and the attenuation term $\kappa(\gamma)$ has an expansion

$$
\kappa(\gamma)=-\frac{\alpha}{\omega_{0}^{2}\left(\omega_{0}^{2}+\alpha^{2}\right)}+O(\gamma)
$$

The poles $\omega_{*}$ and $-\bar{\omega}_{*}$ naturally correspond to the resonant part of the solution,

$$
\begin{aligned}
& y_{\text {res }}(t)=\frac{i}{2 \pi} \gamma^{2} \int\left(\frac{A(\gamma)}{\omega-\omega_{*}}-\frac{\bar{A}(\gamma)}{\omega+\bar{\omega}_{*}}\right) \hat{\jmath}(\omega) e^{-i \omega t} d \omega, \\
& z_{\text {res }}(t)=\frac{i}{2 \pi} \gamma \int\left(\frac{B(\gamma)}{\omega-\omega_{*}}-\frac{\bar{B}(\gamma)}{\omega+\bar{\omega}_{*}}\right) \hat{\jmath}(\omega) e^{-i \omega t} d \omega .
\end{aligned}
$$

The factor of $\gamma^{2}$ in $y_{\text {res }}(t)$ is due to the numerator in (1) when computing the residues. The non-resonant part of the solution comes from the other two poles,

$$
\begin{gathered}
y_{\text {reg }}(t)=\frac{i}{2 \pi} \int\left(\frac{C_{1}(\gamma)}{\omega-\omega_{1}}+\frac{C_{2}(\gamma)}{\omega-\omega_{2}}\right) \hat{\jmath}(\omega) e^{-i \omega t} d \omega, \\
z_{\text {reg }}(t)=\frac{i}{2 \pi} \gamma \int\left(\frac{D_{1}(\gamma)}{\omega-\omega_{1}}+\frac{D_{2}(\gamma)}{\omega-\omega_{2}}\right) \hat{\jmath}(\omega) e^{-i \omega t} d \omega .
\end{gathered}
$$

Powers of $\gamma$ have been extracted from these expressions so that the $\gamma$-dependent residues $A, B, C, D$ are of order 1 . 
The non-resonant part of the solution is associated with direct scattering; particularly, $T(\xi)=y_{\text {reg }}(-\xi / c)$ is the "direct transmission". The factor of $\gamma$ in $z_{\text {res }}(t)$ and $z_{\text {reg }}(t)$ manifests the weak coupling from the string to the resonator, and the $\gamma^{2}$ in $y_{\text {res }}(t)$ manifests the weak coupling back to the string.

The interesting resonant regime occurs when $\hat{\jmath}(\omega)$ is concentrated near the central frequency $\omega_{c}=\operatorname{Re}\left(\omega_{*}\right)$ (and/or $-\omega_{c}$ ) of the resonance (near-monochromatic). In that case the resonator experiences resonant amplitude enhancement- $z_{\text {res }}$ becomes very large despite the factor of $\gamma$-and slow decay. The decay of its energy back into the string is manifest in $y_{\text {res }}(t)$, which grows to order 1 despite the factor of $\gamma^{2}$. This is known as "delayed resonant transmission". The analysis is subtle with regard to the relationships between $\sigma, \gamma$, and $\eta=\bar{\omega}-\omega_{c}$ when all three are small.

\section{NEAR-MONOCHROMATIC FIELDS}

We now take $J(\xi)$ to be a pure oscillation at frequency $\bar{\omega}$ modulated by a broad envelope whose width is controlled by $\sigma^{-1}$ with $\sigma$ being a small parameter,

$$
J(\xi)=g(-\sigma \xi / c) \exp \left(i \frac{\bar{\omega}}{c} \xi\right)
$$

By putting $\xi=x-c t$ and setting $x=0$, one observes that the point-mass on the string experiences this wave temporally as

$$
j(t):=J(-c t)=g(\sigma t) \exp (-i \bar{\omega} t) .
$$

The spectral width of this source field is $\sigma$, which is evident from the Fourier-Laplace transform of $j$,

$$
\hat{\jmath}(\omega)=\int j(t) e^{i \omega t} d t=\frac{1}{\sigma} \hat{g}\left(\frac{\omega-\bar{\omega}}{\sigma}\right) .
$$

\section{A. Resonant amplification}

Given that the forcing frequency $\bar{\omega}$ is close to the central frequency $\omega_{c}$ of the resonance ( $\eta$ is small), $\hat{\jmath}(\omega)$ is concentrated near the complex resonance $\omega_{*}$. Thus just the first term of $z_{\text {res }}(t)$ contributes significant amplification of the resonator. We therefore analyze the quantity

$$
z_{\text {res* }}(t)=\frac{i}{2 \pi} \gamma \int \frac{1}{\omega-\omega_{*}} \hat{\jmath}(\omega) e^{-i \omega t} d \omega
$$

Under the change of variable

$$
\omega=\sigma \phi+\bar{\omega},
$$

and recalling that $\omega_{*}=\omega_{c}-i \kappa \gamma^{2}$ and $\eta=\bar{\omega}-\omega_{c}$, this integral becomes

$$
\begin{aligned}
& z_{\text {res* }}(t)=\frac{\gamma}{\eta+i \kappa \gamma^{2}} e^{-i \bar{\omega} t} \times \\
& \times \underbrace{\frac{i}{2 \pi} \int \frac{\eta+i \kappa \gamma^{2}}{\sigma \phi+\eta+i \kappa \gamma^{2}} \hat{g}(\phi) e^{-i \sigma \phi t} d \phi}_{I(t)},
\end{aligned}
$$

which we now analyze for large time $\left(t>\sigma^{-1}\right)$.

Suppose that the driving term $j(t)$ is "turned on" at $t=0$ and "turned off" at some later time. One might as well let $g(t)$ be supported in the interval $[0,1]$, that is, $g(t)=0$ for $t<0$ and for $t>1$. According to (13), this means that $j(t)$ begins oscillating at time $t=0$ and quits at the large time $t=\sigma^{-1}$. Thus the scatterer is at rest for $t \leq 0$, builds up amplitude during the time interval from $t=0$ to $t=\sigma^{-1}$, and then goes into a superposition of quasi-normal modes (we shall just call them normal modes) corresponding to the four poles for $t>\sigma^{-1}$. We wish to determine the strength of the normal mode corresponding to the resonance at $\omega_{*}$, as it depends on $\gamma, \eta$, and $\sigma$, all being considered to be small.

Given that $g(t)$ is bounded and supported in the time interval $[0,1]$, one has

$$
\begin{array}{ll}
|\hat{g}(\phi)| \leq C e^{|\operatorname{Im} \phi|} / \phi & \text { as } \operatorname{Im} \phi \rightarrow-\infty, \\
|\hat{g}(\phi)| \leq C & \text { as } \operatorname{Im} \phi \rightarrow 0, \\
\hat{g}(\phi) \sim C / \phi & \text { for } \phi \text { real and }|\phi| \rightarrow \infty .
\end{array}
$$

Thus, for time $t>\sigma^{-1}$, the integrand of $I(t)$ in (16) is exponentially decaying as $\operatorname{Im} \phi \rightarrow-\infty$ and the integral can be computed by the residue calculus,

$$
I(t)=\frac{\eta+i \kappa \gamma^{2}}{\sigma} \hat{g}\left(-\frac{\eta+i \kappa \gamma^{2}}{\sigma}\right) e^{-\kappa \gamma^{2} t} e^{i \eta t} .
$$

By defining the function

$$
h(\phi)=\phi e^{i \phi} \hat{g}(-\phi),
$$

one obtains an expression for $z_{\text {res* }}(t)$ for large time,

$$
\begin{array}{r}
z_{\text {res* }}(t)=\frac{\gamma}{\eta+i \kappa \gamma^{2}} h\left(\frac{\eta+i \kappa \gamma^{2}}{\sigma}\right) e^{-i \sigma^{-1} \bar{\omega}} \times \\
\times e^{-i \omega_{c}\left(t-\sigma^{-1}\right)} e^{-\kappa \gamma^{2}\left(t-\sigma^{-1}\right)} \\
\quad\left(\text { for } t>\sigma^{-1}\right) .
\end{array}
$$

Because of the bounds (17) on $|\hat{g}(\phi)|$, the function $h(\phi)$ is bounded by a constant for $\operatorname{Im} \phi<0$.

The expression (19) simplifies further if one considers the regime in which the spectral width of the source field is small compared to the spectral width of the resonance, or $\sigma \ll \gamma^{2}$. In this case, the imaginary part of the argument of $\hat{g}$ becomes unbounded, and one can obtain the asymptotic value

$$
\hat{g}\left(-\frac{\eta+i \kappa \gamma^{2}}{\sigma}\right) \sim g(1) \frac{i \sigma}{\eta+i \kappa \gamma^{2}} e^{-i \eta / \sigma} e^{\kappa \gamma^{2} / \sigma} \quad\left(\sigma \ll \gamma^{2}\right),
$$

uniformly in $\eta / \sigma$. This results in an explicit asymptotic expression for $z_{\text {res* }}(t)$,

$$
\begin{aligned}
z_{\text {res* }}(t) \sim \frac{\gamma}{\eta+i \kappa \gamma^{2}} i g(1) e^{-i \sigma^{-1} \bar{\omega}} \times \\
\times e^{-i \omega_{c}\left(t-\sigma^{-1}\right)} e^{-\kappa \gamma^{2}\left(t-\sigma^{-1}\right)} \\
\left(\text { for } \sigma \ll \gamma^{2} \text { and } t>\sigma^{-1}\right) . \quad(21)
\end{aligned}
$$

We are now in a position to analyze resonant amplification of the spring-mass resonator $z(t)$ in the high-Q $(\gamma$ small $)$ and near-monochromatic ( $\sigma$ small) regime. Equations (19) and (21) for $z_{\text {res* }}(t)$ give an explicit expression for the coefficient of the slowly decaying normal mode $C e^{-i\left(\omega_{c}+\kappa \gamma^{2}\right)\left(t-\sigma^{-1}\right)}$ associated to the complex resonance $\omega_{*}$ for large time $t>\sigma^{-1}$. This is one component of the solution $z(t)$. The other three normal modes in $z(t)$ come from the second term in the integrand of $z_{\text {res }}(t)$ (10) and the non-resonant part of $z(t)$ (12). All of these are of order $\gamma$. Any resonant amplification of the 
spring-mass will come from the prefactors to the exponential $e^{-i\left(\omega_{c}+\kappa \gamma^{2}\right)\left(t-\sigma^{-1}\right)}$ in (19) or (21).

Consider first the regime $\gamma^{2} \ll \sigma$, when the spectral width of the resonance is smaller than the spectral width of the source field. In this case, the imaginary part in the argument of $h$ in (19) tends to zero, and one can use the definition (18) of $h$ and the second bound in (17) to obtain

$$
\begin{aligned}
& z_{\text {res* }}(t) \sim \frac{\gamma}{\sigma} \hat{g}(-\eta / \sigma) e^{-i \omega_{c} t} e^{-\kappa \gamma^{2}\left(t-\sigma^{-1}\right)} \\
& \quad\left(\text { for } \gamma^{2} \ll \sigma \text { and } \eta<C \sigma\right) .
\end{aligned}
$$

The reason for the constraint $\eta<C \sigma$ is that $\hat{g}(-\eta / \sigma)$ vanishes when the argument becomes unbounded. The condition $\eta<C \sigma$ guarantees that $\hat{g}(-\eta / \sigma)$ is not zero (except perhaps for special values of the argument). In this regime, resonant amplification occurs when the additional relation $\sigma \ll \gamma$ is satisfied. Now, if $\gamma^{2} \ll \sigma \ll \eta$, one has $\hat{g}(-\eta / \sigma) \sim C \sigma / \eta$, so that

$$
\begin{aligned}
z_{\text {res* }}(t) \sim C \frac{\gamma}{\eta} e^{-i \omega_{c} t} e^{-\kappa \gamma^{2}\left(t-\sigma^{-1}\right)} & \\
& \left.\quad \text { (for } \gamma^{2} \ll \sigma \ll \eta\right) .
\end{aligned}
$$

Resonant amplification occurs under the additional condition $\eta \ll \gamma$. The summary of the regime $\gamma^{2} \ll \sigma$ is that resonance occurs in the following situations:

$$
\left|z\left(t=\sigma^{-1}\right)\right| \simeq\left\{\begin{array}{ll}
\gamma / \sigma & (\eta<C \sigma) \\
\gamma / \eta & (\sigma \ll \eta \ll \gamma)
\end{array} \quad\left(\gamma^{2} \ll \sigma \ll \gamma\right) .\right.
$$

The symbol " $\simeq$ " indicates that $|z|$ is bounded from above and below by positive multiples of the right-hand side. Of course, as time progresses past $t=\sigma^{-1}, z(t)$ slowly decays by a factor of $e^{-\kappa \gamma^{2}\left(t-\sigma^{-1}\right)}$. Observe that resonant amplification of $z(t)$ in this regime is always less than $C \gamma^{-1}$,

$$
\left|z\left(t=\sigma^{-1}\right)\right| \leq C \gamma^{-1} \quad\left(\gamma^{2} \ll \sigma \ll \gamma\right) .
$$

Consider now the regime $\sigma<C \gamma^{2}$, when the spectral width of the source field is at least as small as the spectral width of the resonance. The nonzero function $h$ is bounded by a constant, so all the important information is in the amplification factor

$$
\mathcal{A}:=\frac{\gamma}{\eta+i \kappa \gamma^{2}} .
$$

This factor depends only on $\gamma$ and $\eta$, not on $\sigma$.

Given that $\left|z_{\text {res* }}(t)\right| \simeq \mathcal{A}$ for $t=\sigma^{-1}$ and that the other normal modes in $z(t)$ are of order $\gamma$, one obtains

$$
\left|z\left(t=\sigma^{-1}\right)\right| \simeq\left\{\begin{array}{ll}
\gamma / \eta & \left(\gamma^{2} \ll \eta<C \gamma\right) \\
\gamma^{-1} & \left(\eta<C \gamma^{2}\right)
\end{array} \quad\left(\sigma<C \gamma^{2}\right) .\right.
$$

To see how $\mathcal{A}$ depends on the asymptotic relation between $\eta$ and $\gamma$, assume a power relation

$$
\eta \sim C \gamma^{p}
$$

If $p<2$, then the denominator of $\mathcal{A}$ is dominated by $\eta$, so that $|\mathcal{A}| \sim C \gamma^{1-p}$ (for a different constant $C$ ). If $p \geq 2$, then the denominator is dominated by $\gamma^{2}$, and one obtains $|\mathcal{A}| \sim C \gamma^{-1}$. In either case, $|\mathcal{A}| \sim C \gamma^{-q}$ with $q=\min \{p-1,1\}$ :

$$
\left|z\left(t=\sigma^{-1}\right)\right| \simeq|\mathcal{A}| \sim C \gamma^{-q}, \quad q=\min \{p-1,1\} .
$$

This relation is depicted in Fig. 4.

If $p<1$, the forcing frequency is sufficiently far from the resonant frequency so that the spring-mass is practically at rest. And if $p>1$, the forcing frequency is close enough to the resonant frequency so that the spring-mass experiences resonant amplification. This amplification does not exceed the inverse power of $\gamma$, which was also the case for the regime $\gamma^{2} \ll \sigma$.

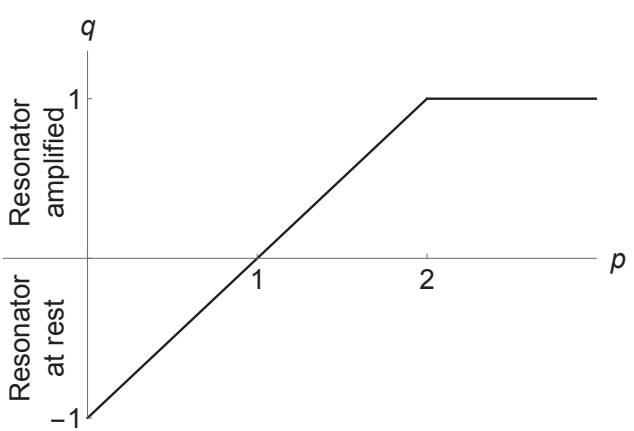

Fig. 4. Resonant amplification of the spring-mass resonator in the regime $\sigma<C \gamma^{2}$, when the spectral width $\sigma$ of the forcing field is comparable to or smaller than the spectral width $\gamma^{2}$ if the resonance. The function $z_{\text {res* }}(t)$ depends on how the detuning of the forcing frequency $\bar{\omega}$ from the resonant frequency $\omega_{c}$ is related to coupling parameter $\gamma$ (the spectral width of the resonance is $\gamma^{2}$ ). Set $\eta=\bar{\omega}-\omega_{c}$, and consider $\gamma, \sigma$, and $\eta$ all to be small. If $\eta$ and $\gamma$ obey a power law $\eta \sim C \gamma^{p}$, then the amplification factor obeys a power law $|\mathcal{A}| \sim C \gamma^{-q}$. The relation between the powers $p$ and $q$ is shown in the graph. For $p<1$ the spring-mass resonator has negligible amplitude-its amplitude becomes zero as $\gamma \rightarrow 0$. For $p>1$, the spring-mass is resonantly excited-its amplitude becomes unbounded as $\gamma \rightarrow 0$.

\section{B. Modified Fano resonance}

The sharp asymmetric anomaly in the monochromatic transmission coefficient depicted in Fig. 2 is typically known as a Fano resonance, in reference to Ugo Fano's famous paper [14]. It is the result of the resonant interaction between a continuum of extended states and a near-bound state. We will analyze how this anomaly is modified when the source field has a finite lifetime but is nearly monochromatic. It degenerates as the source pulse becomes temporally shorter and spectrally wider.

The transmission number $\mathcal{T}$ for a given incident source field $J(\xi)$ is defined as the ratio of total energy transmitted across the resonator to the energy of $J(\xi)$. Because of the relations $j(t)=J(-c t)$ and $y(t)=T(-c t)$ and unitarity (up to a constant) of the Fourier-Laplace transform, one has

$$
\mathcal{T}=\frac{\|T\|^{2}}{\|J\|^{2}}=\frac{\|y\|^{2}}{\|j\|^{2}}=\frac{\|\hat{y}\|^{2}}{\|\hat{\jmath}\|^{2}}
$$

in which $\|f\|=\left(\int|f(s)|^{2} d s\right)^{1 / 2}$, with integration over the real line, denotes the quadratic norm of any function $f$.

In view of $(1,5,14)$, one can define a $\sigma$-dependent transmission coefficient $\mathcal{T}_{\sigma}(\bar{\omega})$ to be the transmission number for a 
near-monochromatic source field $j(t)=g(\sigma t) \exp (-i \bar{\omega} t)$,

$$
\begin{gathered}
\mathcal{T}_{\sigma}(\bar{\omega}):=\frac{\left\|\mathfrak{t}(\omega) \sigma^{-1} \hat{g}\left(\sigma^{-1}(\omega-\bar{\omega})\right)\right\|^{2}}{\left\|\sigma^{-1} \hat{g}\left(\sigma^{-1}(\omega-\bar{\omega})\right)\right\|^{2}} \\
=\frac{\left\|\mathfrak{t}(\omega) \hat{g}\left(\sigma^{-1}(\omega-\bar{\omega})\right)\right\|^{2}}{\sigma\|\hat{g}(\omega)\|^{2}} .
\end{gathered}
$$

(In taking the norms, one integrates over $\omega$ with $\bar{\omega}$ fixed.) If the normalization $\|\hat{g}\|^{2}=1$ is taken, then this coefficient simplifies to a convolution of $\mathfrak{t}(\omega)$ with $\sigma^{-1}\left|\hat{g}\left(\sigma^{-1} \omega\right)\right|^{2}$,

$$
\mathcal{T}_{\sigma}(\omega)=|\mathfrak{t}|^{2} * \frac{1}{\sigma}\left|\hat{g}\left(\frac{\dot{\sigma}}{\sigma}\right)\right|^{2}(\omega)
$$

in which we have integrated over $\omega$ in (27) to compute the norm and then replaced $\bar{\omega}$ with the variable $\omega$. The mollifier $\sigma^{-1}\left|\hat{g}\left(\sigma^{-1} \omega\right)\right|^{2}$ tends to a delta-function as $\sigma$ vanishes, so that

$$
\mathcal{T}_{\sigma}(\omega) \rightarrow|\mathfrak{t}(\omega)|^{2} \quad \text { as } \sigma \rightarrow 0
$$

The near-monochromatic transmission coefficient $\mathcal{T}_{\sigma}(\omega)$ is a regularization of the monochromatic one $\mathcal{T}_{0}(\omega):=|\mathfrak{t}(\omega)|^{2}$.

The width of the Fano resonance in Fig. 2 is of order $\gamma^{2}$. In other words, the transmission coefficient deviates significantly from the "background" direct transmission when $\eta<C \gamma^{2}$. Rigorous analyses are carried out in [12], [13]. Equation (28) shows that the transmission anomaly persists for $\mathcal{T}_{\sigma}(\omega)$ if $\sigma$ is small compared to $\gamma^{2}$ and becomes smoothed out as $\sigma$ becomes relatively large,

$$
\begin{array}{ll}
\sigma \ll \gamma^{2} & \text { sharp anomaly, } \\
\sigma \simeq \gamma^{2} & \text { weak anomaly, } \\
\sigma \gg \gamma^{2} & \text { no anomaly. }
\end{array}
$$

\section{Concluding Discussion}

A resonant version of the classical Lamb model elucidates the fine features of Fano resonance when the source field is nearly monochromatic. A resonant mode weakly coupled to a continuum of radiation states is modeled by a springmass oscillator weakly attached to an infinite string. An exact solution using the Fourier-Laplace transform allows one to cleanly define direct and resonant, or delayed, transmission and reflection. The resonant amplification of the slowly decaying quasi-normal mode associated to the decay of energy out of the resonator depends delicately on the parameters $\gamma$ (coupling to the resonator), $\sigma$ (spectral width of the source field), and $\eta=\bar{\omega}-\omega_{c}$ (difference between source and resonant frequencies).

The resonant amplitude $z(t)$ of the spring-mass oscillator at time $t=\sigma^{-1}$ depends on these three parameters as follows. When $\sigma$ is much larger than $\gamma^{2}$, one has

$$
|z(t)| \simeq \frac{\gamma}{\max \{\sigma, \eta\}} \ll \gamma^{-1} \quad\left(\gamma^{2} \ll \sigma\right),
$$

and when $\sigma$ is not much larger than $\gamma^{2}$, one has

$$
|z(t)| \simeq \frac{\gamma}{\max \left\{\eta, \gamma^{2}\right\}} \quad\left(\sigma<C \gamma^{2}\right) .
$$

After time $t=\sigma^{-1}$, the field decays at a slow exponential rate on the order of $\gamma^{2}$. More elaborate versions of the Lamb model can be devised to elucidate more complex resonance phenomena, such as dependence on the angle of incidence for photonic systems.

\section{REFERENCES}

[1] H. A. Haus and W. Huang, "Coupled-mode theory," Proc. IEEE, vol. 79, no. 10, pp. 1505-1518, 1991.

[2] S. Fan and J. D. Joannopoulos, "Analysis of guided resonances in photonic crystal slabs," Phys. Rev. B, vol. 65, no. 23, pp. 235 112-1-8, Jun 2002.

[3] S. Fan, W. Suh, and J. D. Joannopoulos, "Temporal coupled-mode theory for the Fano resonance in optical resonators," J. Opt. Soc. Am. $A$, vol. 20 , no. 3, pp. 569-572, 2003.

[4] A. Gribovsky and O. Yeliseyev, "Gaussian wave beam scattering on reflect phased antenna array with shorted rectangular cross-section waveguides," Proc. Intl. Conf. on Math. Meth. in EM Theory, Kharkov. IEEE, 2012, pp. 296-299.

[5] N. L. Tsitsas and Valagiannopoulos, "Concentrating the electromagnetic power in a grounded dielectric slab excited by an external gaussian beam," Proc. Intl. Conf. on Math. Meth. in EM Theory, Kharkov. IEEE, 2012, pp. 304-307.

[6] H. Lamb, "On a peculiarity of the wave-system due to free vibrations of a nucleus in an extended medium," Proc. Lond. Math. Soc., vol. XXXII, no. 723, pp. 208-211, 1900.

[7] J. B. Keller and L. L. Bonilla, "Irreversibility and nonrecurrence," $J$. Stat. Phys., vol. 42, no. 5-6, pp. 1115-1125, 1986.

[8] A. I. Komech, "On stabilization of string-nonlinear oscillator interaction," J. Math. Anal. Appl., vol. 196, pp. 384-409, 1995.

[9] A. M. Bloch, P. Hagerty, A. G. Rojo, and M. I. Weinstein, "Gyroscopically stabilized oscillators and heat baths," J. Stat. Phys., vol. 115, pp. 1073-1100, 2004.

[10] S. P. Shipman and S. Venakides, "An exactly solvable model for nonlinear resonant scattering," Nonlinearity, vol. 25, pp. 2473-2501, 2012.

[11] A. Soffer and M. I. Weinstein, "Time-dependent resonance theory," GAFA, Geom. funct. anal., vol. 8, no. 6, pp. 1086-1128, 1998.

[12] S. P. Shipman and S. Venakides, "Resonant transmission near nonrobust periodic slab modes," Phys. Rev. E, vol. 71, no. 1, pp. $026611-$ $1-10,2005$.

[13] S. P. Shipman and A. T. Welters, "Resonant electromagnetic scattering in anisotropic layered media," J. Math. Phys., vol. 54, no. 10, pp. $103511-1-40,2013$.

[14] U. Fano, "Effects of configuration interaction on intensities and phase shifts," Physical Review, vol. 124, no. 6, pp. 1866-1878, 1961. 